\title{
III. Der Aufbau der Jugendstudie Staufen
}

Die Jugendstudie Staufen war von Beginn so angelegt, dass man sich dem zentralen Thema „Freizeitmöglichkeiten von Jugendlichen in Staufen“ aus verschiedenen Blickwinkeln nähern wollte.

Grundbausteine bildeten die am Anfang stehenden Expertengespräche, die nicht nur einen Blick über die in Staufen bereits etablierten Angebote für Jugendliche geben sollten, sondern auch einen Einstieg in das eigentlich Thema. Die Gesamtheit der geschilderten Einschätzungen vermittelten einen ersten Eindruck über die Qualitäten und Defizite der bislang in Staufen geleisteten Jugendarbeit und die Situation der einzelnen Vereine und Institutionen. Daneben stand mit der quantitativen Befragung das einzige repräsentative Element der Studie und gleichzeitig so etwas wie das Kernstück, das es zu ergänzen galt. Ergänzt wurden diese Bausteine durch Gruppendiskussionen, in denen durch die Befragung nur vage erhaltene Antworten nochmals aufgegriffen werden sollten.

Im Verlauf der Studie wurde deutlich, dass speziell die Gruppe der strukturell benachteiligten Jugendlichen, die in Staufen ohnehin bislang kaum in Erscheinung getreten ist, aber sehr wohl existiert, über die Befragung nur sehr schlecht erreicht wurde. Und dabei ging es unter anderem auch gerade um diese Jugendlichen und ihre Bedarfe. Deshalb nutzte man die Offenheit des Studienrahmens, den Verlauf als solchen durch zwei weitere Bausteine zu ergänzen, mit denen man u.a. diese Gruppe zu erreichen hoffte.

Auf die Expertengespräche und die Befragung folgten die Ortsbegehungen und einige Wochen später eine Videodokumentation. Abgerundet wurden alle Bausteine durch die zeitlich nah an der Umsetzungsphase liegenden Gruppendiskussionen, die neben der bereits erwähnten Klärung ungenauer Aussagen auch noch einmal ganz aktuelle Themen aufgreifen und beleuchten sollten. Etwa zwei Wochen nach der letzten Gruppendiskussion fand das 1. Staufener Jugendforum statt, das den Beginn der Transformationsphase bildete.

Auf die Vorbereitung, den Verlauf und die Erfahrungen, die mit den einzelnen Bausteinen gemacht wurden, wird im Folgenden eingegangen. 